\title{
Ações de educação em saúde sobre sífilis com adolescentes: revisão integrativa
}

\author{
Health education actions on syphilis with adolescentes: integrative review \\ Acciones de educación en salud sobre sífilis con adolescentes: revisión integradora
}

\author{
Débora Campos Soares Araújo \\ ORCID: https://orcid.org/0000-0003-3928-0129 \\ Universidade Federal de São João del-Rei, Brasil \\ E-mail: dehhsoares@hotmail.com \\ Daniela Aparecida de Faria \\ ORCID: https://orcid.org/0000-0001-8938-9371 \\ Universidade Federal de São João del-Rei, Brasil \\ E-mail: danielaffisio@hotmail.com \\ Alisson Araújo \\ ORCID: https://orcid.org/0000-0002-4623-3745 \\ Universidade Federal de São João del-Rei, Brasil \\ E-mail: alissonenf@hotmail.com
}

\begin{abstract}
Resumo
Este estudo teve por objetivo analisar as evidências científicas sobre as intervenções de educação em saúde de sífilis em adolescentes. Revisão integrativa realizada nas bases de dados BVS, PubMED, Science Direct e Web of Science, sem limite de tempo e sem restrição quanto a data de início da coleta, uma vez que o objetivo foi recuperar o máximo de artigos possíveis e que descrevessem ações educativas a nível de prevenção da sífilis em adolescentes. Inicialmente foram identificados 1.377 artigos, mas apenas nove atenderam aos critérios de elegibilidade. A literatura mostrou que intervenções educacionais como rodas de conversa, jogos e oficinas sobre métodos contraceptivos foram eficazes para problematizar de forma ativa a participação dos adolescentes bem como uma importante oportunidade de reflexão e discussão, ampliando o campo de conhecimento deles sobre esses temas. Os jogos educativos foram o recurso de maior ocorrência encontrado nos estudos e com resultados positivos. Ressalta-se que a escola faz-se um cenário favorável nesse diálogo, uma vez que, por se tratar de ambiente do cotidiano dos adolescentes, onde permanecem a maior parte do dia, sentem-se seguros para expressar suas dúvidas, medos e sentimentos. Concluiu-se que existe uma lacuna de estudos primários na temática envolvida principalmente no público adolescente. Destacando-se desta forma, a real necessidade de desenvolvimento e aplicação prática de estratégias de educação em saúde na prevenção de sífilis na adolescência.
\end{abstract}

Palavras-chave: Educação em saúde; Adolescente; Sífilis.

\begin{abstract}
This study aimed to analyze the scientific evidence on health education interventions for syphilis in adolescents. Integrative review performed in VHL, PubMED, Science Direct and Web of Science databases, no time limit and no restriction on the date when collection started, since the objective was to retrieve as many articles as possible and describing educational actions regarding the prevention of syphilis in adolescents. 1,377 articles were initially identified, but only nine met the eligibility criteria. The literature has shown that educational interventions such as conversation circles, games and workshops on contraceptive methods were effective in actively questioning the participation of adolescents, in addition to being an important opportunity for reflection and discussion, expanding their field of knowledge on these topics. Educational games were the resource most found in studies and with positive results. It is noteworthy that the school is a favorable environment in this dialogue, since, as it is the daily environment of adolescents, where they spend most of the day, they feel safe to express their doubts, fears and feelings. It was concluded that there is a gap in primary studies on the subject that mainly involve adolescents. This highlights the real need for the development and practical application of health education strategies for the prevention of syphilis in adolescence. Keywords: Health education; Adolescent; Syphilis.
\end{abstract}

\section{Resumen}

Este estudio tuvo como objetivo analizar la evidencia científica sobre las intervenciones de educación en salud para la sífilis en adolescentes. Revisión integrativa realizada en las bases de datos VHL, PubMED, Science Direct y Web of Science, sin límite de tiempo y sin restricción en la fecha de inicio de la recolección, ya que el objetivo era recuperar la mayor cantidad de artículos posible y describir acciones educativas sobre la prevención de la sífilis en adolescentes. Inicialmente se identificaron 1.377 artículos, pero solo nueve cumplieron los criterios de elegibilidad. La literatura ha 
demostrado que intervenciones educativas como círculos de conversación, juegos y talleres sobre métodos anticonceptivos fueron efectivas en cuestionar activamente la participación de los adolescentes, así como una importante oportunidad de reflexión y discusión, ampliando su campo de conocimiento sobre estos temas. Los juegos educativos fueron el recurso más frecuente encontrado en los estudios y con resultados positivos. Es de destacar que la escuela es un escenario propicio en este diálogo, ya que, al ser el entorno cotidiano de los adolescentes, donde pasan la mayor parte del día, se sienten seguros para expresar sus dudas, miedos y sentimientos. Se concluyó que existe un vacío en los estudios primarios sobre el tema que involucran principalmente a adolescentes. Esto resalta la necesidad real del desarrollo y la aplicación práctica de estrategias de educación en salud para la prevención de la sífilis en la adolescencia.

Palabras clave: Educación para la salud; Adolescente; Sífilis.

\section{Introdução}

As infecções sexualmente transmissíveis (IST’s) são consideradas mundialmente um problema de saúde pública e encontram-se entre as principais doenças transmissíveis mais comuns (Brasil, 2020). Dentre estas, a sífilis, apesar de ser uma infecção antiga, com diagnóstico e tratamento consolidado, ainda se constitui um grave problema de Saúde Pública por sua magnitude e epidemiologia mundial (World Health Organization, 2015).

Estima-se que, no mundo, ocorram anualmente cerca de 12 milhões de novos casos de sífilis (WHO, 2016). No Guideline de tratamento para sífilis da WHO (2016) destaca que dos novos casos anuais de sífilis no mundo, 1,5 a 1,85 milhões são de gestantes, e que 50\% delas têm filhos com resultados adversos devido às consequências da doença. A World Health Organization (WHO, 2015) adverte sobre os impactos negativos diretos da sífilis, como por exemplo na saúde reprodutiva, infantil, infertilidade, complicações na gravidez, morbimortalidade nos desfechos materno-fetais bem como maior propensão e facilitação no desenvolvimento e transmissão do vírus da imunodeficiência humana (HIV).

A WHO (2015) instituiu a sífilis como prioridade para implementação de ações de prevenção e controle das IST’s nos anos de 2016 a 2021 em conformidade com a Agenda 2030 para o Desenvolvimento Sustentável, no qual lançou o plano estratégico mundial (2016 a 2021) sobre a abordagem nas IST's que tem como meta na redução de $90 \%$ da incidência de sífilis até o ano 2030. Da mesma forma, a Organização Pan-Americana de Saúde (OPAS, 2016) publicou um plano de ação para prevenção e controle de HIV/IST, com o objetivo de acelerar a eliminação das epidemias de HIV e IST na Região das Américas até 2030 .

Nesse aspecto, cada país busca definir intervenções baseadas nos aspectos epidemiológicos e sociais locais. No Brasil, foi implementada a agenda estratégica para redução da transmissão da sífilis a partir de 2016 (ano em que foi anunciada a epidemia de sífilis pelo Ministério da Saúde) sempre alinhada com as ações da OPAS e WHO (Brasil, 2017).

No Brasil, a situação da sífilis não é diferente dos outros países, os números de casos da infecção são alarmantes e preocupantes. De acordo com o Novo Boletim Epidemiológico do Ministério da Saúde (Brasil, 2020) observa-se que a sífilis adquirida, em 2019, teve uma taxa de detecção de 72,8 casos por 100.000 habitantes. Também em 2019, a taxa de detecção de sífilis em gestantes foi de 20,8/1.000 nascidos vivos; a taxa de incidência de sífilis congênita, de 8,2/1.000 nascidos vivos; e a taxa de mortalidade por sífilis congênita, de 5,9/100.000 nascidos vivos. A sífilis congênita no Brasil é um agravo de notificação compulsória desde 1986 (Luppi et al., 2018), já a sífilis adquirida, é um agravo de notificação compulsória desde 2010.

Ultimamente no Brasil, tem chamado atenção o aumento da prevalência de sífilis em indivíduos em uma fase da vida bastante distinta: a adolescência. O Ministério da Saúde (Brasil, 2018) em conformidade à Organização Mundial da Saúde delimita a adolescência como o período entre os 10 a 19 anos. A adolescência é uma fase do ciclo vital no qual ocorrem inúmeras transformações biopsicossociais. O adolescente manifesta sua sexualidade por meio de diferentes sensações e desejos corporais ainda desconhecidos, por novas necessidades de relacionamento interpessoais e influência de pares, além de inúmeras curiosidades e descobertas. Nesse sentido, o comportamento e atitude dos adolescentes encontra-se em processo de formação e consolidação, tornando desta forma esse segmento populacional vulnerável (Brasil, 2018). 
Diante dos expressivos números da epidemiologia mundial da sífilis e em especial no acometimento na fase da adolescência, reforça-se a relevância desse agravo como problema de saúde pública. É urgente a necessidade de articular respostas resolutivas e eficazes, tanto no tratamento quanto na prevenção da doença, onde esta última através de estratégias no campo da educação em saúde possa de alguma forma produzir impacto satisfatório de redução dos números nesse público. Desta forma, justifica-se a presente revisão, com vistas a minimamente identificar e fornecer subsídios para o aprimoramento de estratégias em educação em saúde da sífilis junto a esse grupo, tendo como principal objetivo analisar as evidências disponíveis sobre as intervenções de educação em saúde na prevenção de sífilis em adolescentes.

\section{Metodologia}

Trata-se de uma revisão integrativa da literatura, um tipo de pesquisa que baseia-se na busca de trabalhos relevantes sobre um determinado assunto, que possibilita identificar lacunas que possam ser preenchidas com a realização de outros estudos (Sousa, et al., 2020). Este desenho de pesquisa proporciona uma síntese e avaliação crítica sobre a temática investigada, proporcionando uma organização atual do conhecimento e reflexões para a implementação de novas intervenções na prática (Mowbray, Wilkinson, Tse, 2015).

A revisão integrativa seguiu as seguintes etapas: a) identificação do tema e formulação da pergunta da pesquisa; b) estabelecimento de critérios de inclusão e exclusão dos estudos para amostragem; c) coleta dos dados que foram extraídos dos estudos; d) análise crítica dos estudos selecionados; e) interpretação dos resultados; f) apresentação da síntese estabelecida e revisão dos conteúdos (Mendes, Silveira, Galvão, 2008; Honório \& Santiago Júnior, 2021).

Para atingir o objetivo proposto, foi definida a seguinte pergunta norteadora: Quais as evidências científicas sobre intervenções de educação em saúde na prevenção de sífilis em adolescentes? A pergunta do estudo foi elaborada através da estratégia PICO, no qual considerou-se: População: adolescentes de 10 a 19 anos; Intervenção: estratégias de educação em saúde para prevenção sífilis; Comparação: não se aplica; “Outcomes”: prevenção da sífilis em adolescentes.

A busca foi realizada por meio dos artigos publicados e indexados em banco de dados eletrônicos da BVS (Biblioteca Virtual em Saúde), PubMed (US National Library of Medicine National Institutes of Health), Science Direct e Web of Science. Os artigos comuns às bases de dados pesquisadas foram considerados apenas uma vez. Para a busca nas bases de dados, utilizaram-se os descritores controlados contidos nos Descritores em Ciência da Saúde (DeCS) da BVS e MeSH (Medical Subject Headings), das palavras-chave: "Health Education", "Syphilis", "Adolescent" e os operadores booleanos associados com os operadores booleanos AND e OR. As estratégias completas utilizadas em cada bases de dados estão apresentadas no Quadro 1.

Foram considerados elegíveis artigos publicados na íntegra, que contemplassem: a temática, nos idiomas português e inglês; a população adolescente na amostra; metodologias de educação em saúde abordando sífilis, estudos encontrados na literatura científica sem limite de tempo e sem restrição quanto a data de início da coleta, uma vez que o objetivo foi recuperar o máximo de artigos possíveis e que descrevessem ações educativas a nível de prevenção da sífilis em adolescentes. Foram excluídos os artigos de revisão, as cartas ao editor, teses e dissertações, as notícias e comentários. O levantamento bibliográfico foi realizado nos meses de maio e junho de 2021. 
Quadro 1: Estratégias de busca utilizadas nas bases de dados PubMed, BVS, Science Direct e Web of Science. Divinópolis, MG, 2021.

\begin{tabular}{|l|l|}
\hline Base de dados & Estratégia de busca \\
\hline PubMED & $\begin{array}{l}\text { (("health education"[MeSH Terms] OR ("health"[All Fields] AND "education"[All Fields]) } \\
\text { OR "health education"[All Fields]) AND ("adolescent"[MeSH Terms] OR "adolescent"[All } \\
\text { Fields])) AND ("syphilis"[MeSH Terms] OR "syphilis"[All Fields]) }\end{array}$ \\
\hline BVS & $\begin{array}{l}\text { (MH:"Educação em saúde" OR (Educação) OR (Health Education) OR (Educación en Salud) } \\
\text { OR MH: I02.233.332 OR MH: N02.421.726.407 OR MH: SP2.021.172 OR MH: } \\
\text { SP8.946.234.289) AND (MH:"Sífilis" OR (Sífilis) OR (Syphilis) OR (Sífilis) OR MH: } \\
\text { C01.150.252.400.794.840.500 OR MH: C01.150.252.400.840.500 OR MH: } \\
\text { C01.150.252.734.859 OR MH: C12.294.668.281.859 MH: C13.351.500.711.281.859\$) AND } \\
\text { (MH:"Adolescente" OR (Adolescente) OR (Adolescent) OR (Adolescente) OR MH: } \\
\text { M01.060.057\$) }\end{array}$ \\
\hline Science Direct & \begin{tabular}{l} 
("Health Education") AND ("adolescent") AND ("Syphilis") \\
\hline Web of Science
\end{tabular} \\
\hline
\end{tabular}

Fonte: Autores.

Após a elaboração da estratégia de busca, os artigos foram enviados para a plataforma Rayyan (2021) para retirada de duplicação e leitura dos estudos.

Analisaram-se publicações pesquisadas crítica e independentemente por dois autores D.F. e D.A. As indecisões referentes à seleção dos artigos foram avaliadas em plenária com a participação de um terceiro pesquisador A.A., até se obter concordância. Para minimizar os riscos vieses, a busca, a avaliação e a seleção dos estudos se deram por três revisores, e ao final foi realizada uma discussão para consenso dos artigos a serem incluídos na revisão.

O processo de seleção dos artigos seguiu o Protocolo PRISMA (Preferred Reporting Items for Systematic Reviews and Meta-Analyses), para o processo de identificação, seleção, elegibilidade e inclusão dos estudos. Todos os estudos selecionados foram lidos na íntegra.

Para classificar o nível de evidência dos artigos, foi utilizada a categorização da Agency for Health care Research and Quality (2021) segundo a qual o nível 1 é considerado o de maior força de evidência, no qual são incluídas as metanálises de múltiplos estudos controlados. Projetos individuais com desenho experimental, como os ensaios clínicos aleatórios, são considerados de nível 2. Estudos de coorte, caso-controle e quase experimentais, como estudos não randomizados, são classificados como nível 3. Estudos com desenho não experimental, como os transversais, recebem o nível de evidência 4. Relatórios de caso são considerados nível 5, e opiniões de autoridades respeitáveis baseadas na competência clínica ou opinião de comitês de especialistas e interpretações de informações não baseadas em pesquisas estão no nível 6.

A seleção ocorreu por meio de leitura de títulos, resumos e quando necessária, a leitura íntegra dos textos como forma de selecioná-los de acordo com os critérios de inclusão e exclusão.

No processo de análise foram coletados dados referentes ao periódico (ano de publicação, autores e continente); e ao estudo (delineamento do estudo, nível de evidência, objetivo, instrumento utilizado, principais resultados). 
Figura 1: Fluxograma Preferred Reporting Items for Systematic Reviews and Meta-Analyses (PRISMA) sobre a seleção dos estudos.

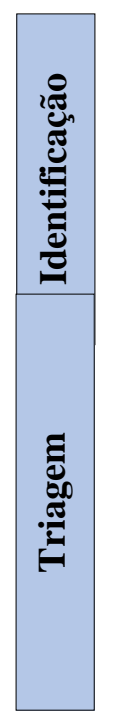

Estudos identificados nas bases de dados eletrônicos

$$
(\mathrm{n}=1.373)
$$

Artigos após retirada da duplicação

$$
(\mathrm{n}=795)
$$
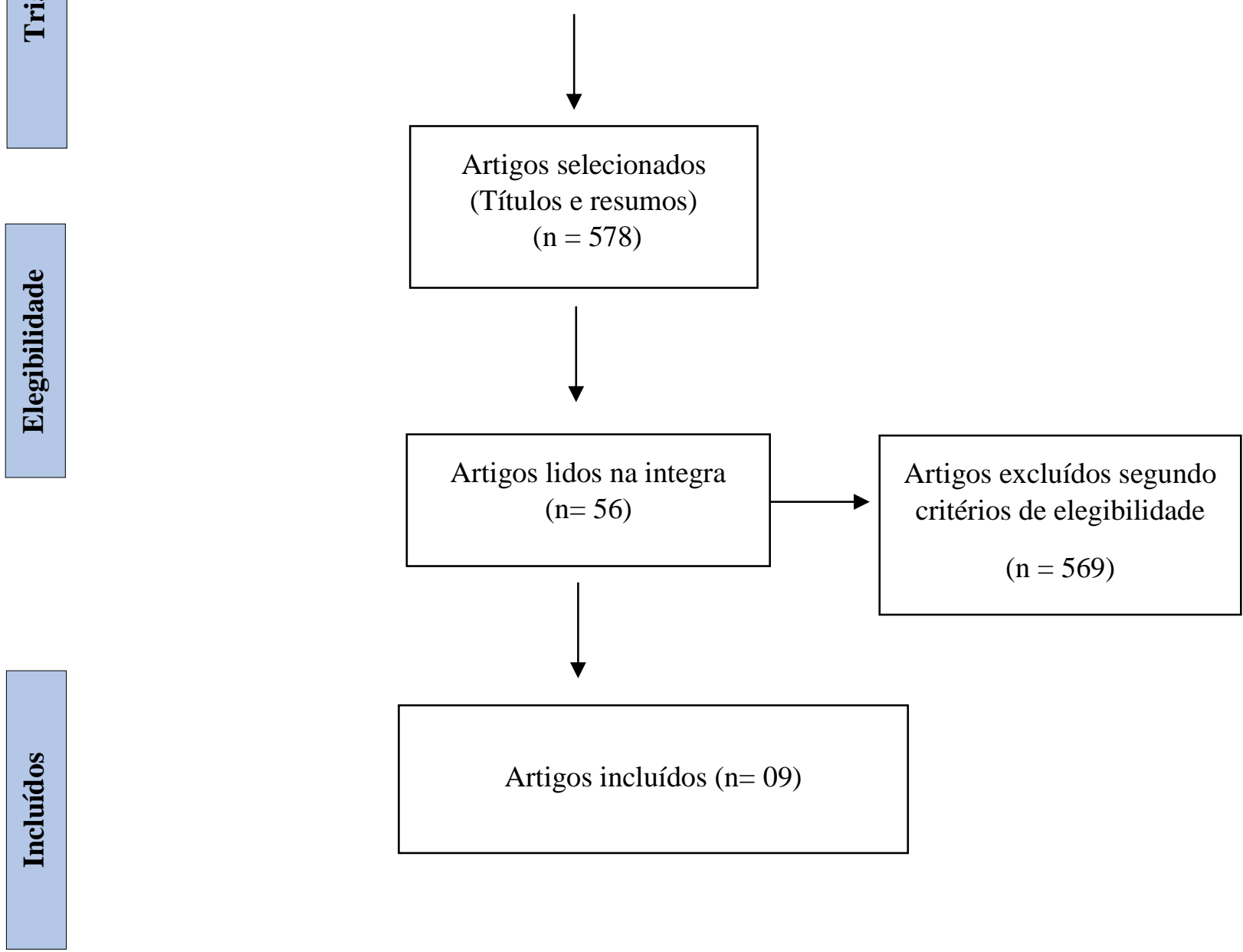

Artigos incluídos (n=09)

From:Libertati, et al. The PRISMA statement for reporting systematic reviews and meta-analyses of studies that evaluate healthcare interventions: explanation and elaboration. 2009. BMJ, v. 339. doi: https://doi.org/10.1136/bmj.b2700. Fonte: Autores. 
Quadro 2: Informações e características dos estudos incluídos na revisão (n=09).

\begin{tabular}{|c|c|c|c|c|}
\hline $\begin{array}{c}\text { Ano de } \\
\text { publicação/ } \\
\text { Continente }\end{array}$ & Autores/ Objetivo & $\begin{array}{c}\text { Delineamento do estudo/ } \\
\text { Nível de evidência/ } \\
\text { Amostra }\end{array}$ & Instrumento e metodologia & Principais resultados \\
\hline $\begin{array}{l}2008 \\
\text { América do } \\
\text { Sul }\end{array}$ & $\begin{array}{l}\text { Bezerra, E.P., Pinheiro, } \\
\text { P.N.C., Barroso, M.G.T. } \\
\text { Investigar a sexualidade } \\
\text { das adolescentes através } \\
\text { da ação educativa do } \\
\text { enfermeiro na prevenção } \\
\text { de doenças sexualmente } \\
\text { transmissíveis }\end{array}$ & $\begin{array}{c}\text { Estudo transversal } \\
\text { Nível } 4 \\
10 \text { meninas de } 14 \text { e } 16 \text { anos }\end{array}$ & $\begin{array}{l}\text { Adotou-se como método o Círculo de Cultura, que } \\
\text { foi formado por cinco encontros. Utilizaram-se a } \\
\text { observação e a observação participante para coleta } \\
\text { dos dados, que foram registrados no diário de } \\
\text { campo. }\end{array}$ & $\begin{array}{l}\text { Apontou que a execução do Círculo de Cultura permitiu } \\
\text { que as meninas explorassem e discutissem sobre diversos } \\
\text { temas que englobavam sua sexualidade, e que era um } \\
\text { momento para atividades de educação em saúde com a } \\
\text { meta de isentá-las de riscos. }\end{array}$ \\
\hline $\begin{array}{l}2009 \\
\text { América do } \\
\text { Sul }\end{array}$ & $\begin{array}{l}\text { Gubert, F.A., et al. } \\
\text { Discute a utilização de } \\
\text { ferramentas educativas } \\
\text { como técnica de educação } \\
\text { em saúde r com } \\
\text { adolescentes no cenário } \\
\text { escolar. }\end{array}$ & $\begin{array}{c}\text { Estudo transversal } \\
\text { Nível } 4 \\
\text { Adolescentes } 14 \text { a } 18 \text { anos }\end{array}$ & $\begin{array}{l}\text { Foram realizadas oficinas educativas (quatro) com } \\
30 \text { adolescentes. As oficinas tiveram como propósito } \\
\text { estimular o pensamento e comportamento dos } \\
\text { participantes a respeito dos temas: sexualidade, } \\
\text { gênero, DST/AIDS e métodos contraceptivos. } \\
\text { Estudo do tipo pesquisa-ação, de abordagem } \\
\text { qualitativa, sustentado no Modelo Pedagógico de } \\
\text { Paulo Freire. }\end{array}$ & $\begin{array}{l}\text { Os resultados mostraram falhas no conhecimento } \\
\text { relacionadas aos meios de contaminação pelas DST e } \\
\text { utilização correta de métodos contraceptivos. Também faz } \\
\text { referência às questões de gênero que incutem no bem-estar } \\
\text { dos jovens estudados. A utilização de ferramentas } \\
\text { educativas estimulou entre os adolescentes uma reflexão a a } \\
\text { respeito da prática da saúde sexual e reprodutiva a partir } \\
\text { das fragilidades percebidas. Recomendou-se através dos } \\
\text { achados, que as dinâmicas de prevenção às DST/AIDS no } \\
\text { cenário escolar, quebrem a visão heterossexista, normativa } \\
\text { e biologicista. }\end{array}$ \\
\hline $\begin{array}{l}2010 \\
\text { América do } \\
\quad \text { Sul }\end{array}$ & $\begin{array}{l}\text { Barbosa, S.M., et al. } \\
\text { Expor a utilização de } \\
\text { jogos educativos como } \\
\text { método de educação em } \\
\text { saúde para adolescentes. } \\
\text { Estudo exploratório } \\
\text { descritivo realizado em } \\
\text { uma escola pública de } \\
\text { Fortaleza-CE. }\end{array}$ & $\begin{array}{c}\text { Estudo Transversal } \\
\text { Nível } 4 \\
\text { Adolescentes de } 14 \text { e } 19 \text { anos }\end{array}$ & $\begin{array}{l}\text { Para as ações educativas, foi usado um jogo } \\
\text { semelhante ao dominó, contendo } 30 \text { peças com } \\
\text { indagações e respostas. Planejado para ser realizado } \\
\text { com no mínimo duas pessoas. O jogo, abordou } \\
\text { assuntos referentes à sexualidade dos adolescentes: } \\
\text { conceituação de anatomia feminina e masculina, } \\
\text { puberdade e adolescência, sexo/sexualidade, } \\
\text { DST/HIV/AIDS, gravidez e métodos preventivos. }\end{array}$ & $\begin{array}{l}\text { Realizada a aplicação do jogo e do pós-teste, verificou-se } \\
\text { a eficiência da ação educativa participativa. Os dados } \\
\text { mostraram os alunos, em sua maioria, compreenderam os } \\
\text { conteúdos debatidos pelo grupo. A utilização do jogo } \\
\text { educativo foi uma tentativa bem sucedida por ter } \\
\text { proporcionado o desempenho do ato educativo por } \\
\text { intermédio da junção de informações, discussões, } \\
\text { reflexões, interação e participação grupal, na qual os } \\
\text { adolescentes tiveram a oportunidade de sanar seus } \\
\text { questionamentos, completar as brechas no conhecimento } \\
\text { em dúvidas relacionadas a sexualidade e prevenção de } \\
\text { DST e AIDS e comunicar-se de forma desinibida, } \\
\text { possibilitando a interação de todos durante a aquisição de } \\
\text { conhecimento. }\end{array}$ \\
\hline 2012 & 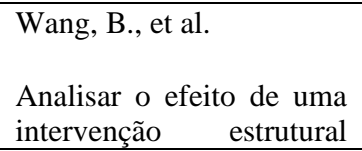 & Estudo transversal & $\begin{array}{l}\text { Programas de rotina de divulgação da saúde } \\
\text { (fornecimento de preservativos e materiais de } \\
\text { educação em saúde); prevenção estrutural } \\
\text { (promoção da saúde na televisão e no jornal para }\end{array}$ & $\begin{array}{l}\text { Um total de } 8.275 \text { mulheres foram elegíveis, e } 3597 \\
\text { mulheres matriculadas }(\mathrm{n}=2011 \text { no braço de controle, } \mathrm{n}= \\
1586 \text { no braço de intervenção) no estudo. A duração média } \\
\text { do seguimento foi de } 375 \text { dias (intervalo interquartil, } 267-\end{array}$ \\
\hline
\end{tabular}




\begin{tabular}{|c|c|c|c|c|}
\hline & $\begin{array}{l}\text { abrangente integrada } \\
\text { sobre sífilis incidente e } \\
\text { infecção pelo HIV em } 6 \\
\text { cidades do sul da China, } \\
\text { tendo como público alvo, } \\
\text { mulheres profissionais do } \\
\text { sexo. }\end{array}$ & $\begin{array}{l}\text { Adolescentes do sexo com } \\
\text { idade } \geq 15 \text { anos }\end{array}$ & $\begin{array}{l}\text { aumentar a conscientização e comportamentos de } \\
\text { busca de saúde; triagem regular de sífilis; e maior } \\
\text { conexão entre serviços de IST de divulgação e } \\
\text { clínica por meio de serviços de referência } \\
\text { aprimorados). }\end{array}$ & $\begin{array}{l}\text { 475). A densidade de incidência de sífilis no grupo de } \\
\text { intervenção foi reduzida em } 70 \% \text { (intervalo de confiança } \\
\text { de } 95 \% \text {, 53\%-81\%) em comparação com a incidência no } \\
\text { braço de controle. Os benefícios de intervenção de } \\
\text { prevenção da sífilis foram robustos entre as mulheres } \\
\text { profissionais do sexo em locais de baixa escolaridade, } \\
\text { indivíduos com menor escolaridade, migrantes e mulheres } \\
\text { que não relataram a utilização de preservativo durante o } \\
\text { último episódio de sexo. }\end{array}$ \\
\hline $\begin{array}{l}2013 \\
\text { América do } \\
\text { Sul }\end{array}$ & $\begin{array}{l}\text { Oliveira, K.N.S, et al. } \\
\text { Investigar o conhecimento } \\
\text { de estudantes de escolas } \\
\text { públicas relacionadas a } \\
\text { sexualidade, métodos } \\
\text { contraceptivos e DST }\end{array}$ & $\begin{array}{c}\text { Estudo transversal } \\
\text { Nível } 4 \\
\text { Adolescentes e jovens de } 10 \mathrm{a} \\
24 \text { anos }\end{array}$ & $\begin{array}{l}\text { Questionário (pré-teste) } \text { para identificar } 0 \\
\text { conhecimento prévio dos adolescentes. Após a } \\
\text { conclusão das oficinas, aplicou-se o mesmo } \\
\text { questionário (pós-teste) }\end{array}$ & $\begin{array}{l}\text { Foram realizadas em três encontros, para cada turma, } \\
\text { totalizando } 12 \text { horas, abordando práticas de educação em } \\
\text { saúde sexual, utilizando diversas técnicas que facilitam o } \\
\text { desenvolvimento de metodologia participativa. Entre elas, } \\
\text { destacam-se: apresentação de peça teatral, um jogo sobre } \\
\text { os métodos contraceptivos e, ao final, ocorreu uma } \\
\text { dinâmica em grupo, em que foram trabalhados os } \\
\text { principais questionamentos, apresentados nas ações do } \\
\text { projeto, sobre os aspectos do desenvolvimento puberal e } \\
\text { as implicações da sexualidade. }\end{array}$ \\
\hline $\begin{array}{l}2014 \\
\text { América do } \\
\text { Sul }\end{array}$ & $\begin{array}{l}\text { Andrade, L.D.F, et al. } \\
\text { Relatar as experiências } \\
\text { vivenciadas durante as } \\
\text { ações educativas } \\
\text { desenvolvidas para jovens } \\
\text { do } 8^{\circ} \text { e } 9^{\circ} \text { ano de uma } \\
\text { escola pública sobre a } \\
\text { temática sífilis, suas } \\
\text { formas de prevenção, } \\
\text { diagnóstico e tratamento }\end{array}$ & $\begin{array}{c}\text { Relato de experiência } \\
\text { Nível } 5 \\
\text { Adolescentes de } 13 \text { a } 19 \text { anos }\end{array}$ & Roda de conversa, peças de teatro. & $\begin{array}{l}\text { O uso das metodologias lúdicas facilitaram a } \\
\text { problematização na temática e na troca de experiência. } \\
\text { Observou-se que as metodologias problematizadoras } \\
\text { como as rodas de conversa, como também as que } \\
\text { demandam métodos lúdicos facilitaram a troca de } \\
\text { experiência entre a equipe do projeto e os adolescentes. No } \\
\text { entanto, ainda há necessidade de ampliar a divulgação das } \\
\text { DSTs entre os jovens, bem como capacitar os professores } \\
\text { do ensino fundamental para estarem abordando temáticas } \\
\text { relativas ao cuidado em saúde com seus alunos. }\end{array}$ \\
\hline $\begin{array}{l}2019 \\
\text { Ásia }\end{array}$ & $\begin{array}{l}\text { Reduzir as infecções por } \\
\text { HIV /DST entre mulheres } \\
\text { trabalhadoras do sexo } \\
\text { (FSWs) de baixo custo por } \\
\text { meio de um ensaio de } \\
\text { intervenção randomizada } \\
\text { com base na comunidade } \\
\text { de pares combinados } \\
\text { realizado em } 12 \text { cidades } \\
\text { em três províncias da } \\
\text { China. }\end{array}$ & $\begin{array}{c}\text { Estudo randomizado } \\
\text { Nível } 4 \\
\text { Adolescentes, jovens e adultos } \\
\text { de } 18 \text { a } 60 \text { anos }\end{array}$ & $\begin{array}{l}\text { Entrevista, triagem. O pacote de intervenção incluiu } \\
\text { promoção de preservativos, teste de HIV e sífilis, } \\
\text { reembolso para tratamento de infecção por sífilis e } \\
\text { terapia anti-retroviral. Receberam atividades de } \\
\text { extensão de rotina, que incluíam distribuição de } \\
\text { preservativos, teste anual de HIV / sífilis e } \\
\text { encaminhamento para infecção por HIV / DST }\end{array}$ & $\begin{array}{l}\text { Um total de } 1.024 \text { FSWs elegíveis foram inscritos na } \\
\text { pesquisa de linha de base e } 843 \text { no acompanhamento. Os } \\
\text { resultados mostraram que a infecção por sífilis foi } \\
\text { reduzida em } 49 \% \text { no grupo de intervenção em comparação } \\
\text { com o padrão atual do grupo de cuidados }(\mathrm{P}=0,0378 \text {, OR } \\
=0,51,95 \% \text { CI: } 0,27-0,96) \text {. Este estudo demonstrou que } \\
\text { intervenções abrangentes podem levar a declínios } \\
\text { significativos na infecção por sífilis entre FSWs de baixo } \\
\text { nível. A integração de medidas de intervenção } \\
\text { comportamentais e biomédicas deve ser considerada ao } \\
\text { desenvolver programas para FSWs de baixo custo. }\end{array}$ \\
\hline
\end{tabular}


Research, Society and Development, v. 10, n. 12, e545101220577, 2021

(CC BY 4.0) | ISSN 2525-3409 | DOI: http://dx.doi.org/10.33448/rsd-v10i12.20577

\begin{tabular}{|c|c|c|c|c|}
\hline $\begin{array}{l}\text { América do } \\
\text { Sul }\end{array}$ & $\begin{array}{l}\text { Fonseca, A.D.M., et al. } \\
\text { Relatar uma ação } \\
\text { educativa por meio de } \\
\text { tecnologias educacionais } \\
\text { sobre sífilis adquirida para } \\
\text { estudantes de uma escola } \\
\text { estadual do Pará }\end{array}$ & $\begin{array}{c}\text { Relato de experiência } \\
\text { Nível } 5 \\
\text { Adolescentes de } 15 \text { a } 17 \text { anos }\end{array}$ & $\begin{array}{l}\text { A construção da atividade foi baseada em três } \\
\text { momentos. No primeiro momento foi elaborado e } \\
\text { distribuição de um folder educativo para os alunos, } \\
\text { no segundo momento foi aplicado uma tecnologia } \\
\text { adaptada para a realidade dos estudantes elaborada } \\
\text { pelos autores, denominado jogo da memória e, no } \\
\text { terceiro momento foi utilizado como atividade } \\
\text { complementar a dinâmica dos balões onde } \\
\text { estimulou-se uma competição de conhecimentos. }\end{array}$ & $\begin{array}{l}\text { Foi observado que os alunos, em sua maioria, } \\
\text { desconheciam a doença como sexualmente transmissível } \\
\text { assim como sua prevenção e a existência do teste rápido } \\
\text { como forma de diagnóstico, mas já tinham ouvido falar e } \\
\text { sabiam que a sífilis tem cura. Ressaltou a relevância de } \\
\text { ações que visem à prevenção da doença através de } \\
\text { tecnologias inovadoras. Sendo de suma importância para } \\
\text { que o processo de ensino-aprendizagem se consolide. }\end{array}$ \\
\hline $\begin{array}{l}2021 \\
\text { Ásia }\end{array}$ & $\begin{array}{l}\text { Xing, M.A., Yang, Y., } \\
\text { Chow, K.M., Zang, Y. } \\
\text { Investigar a eficácia de um } \\
\text { programa interativo de } \\
\text { educação em saúde sexual } \\
\text { e reprodutiva em aspectos } \\
\text { de conhecimento, atitudes } \\
\text { e auto eficácia entre } \\
\text { adolescentes. }\end{array}$ & $\begin{array}{l}\text { Estudo randomizado caso } \\
\text { controle } \\
\text { Nível } 1 \\
\text { Adolescentes de } 10 \text { a } 19 \text { anos }\end{array}$ & $\begin{array}{l}\text { Ficha de informações dados sociodemográficos, } \\
\text { Escala de conhecimento sexual, Questionário de } \\
\text { atitudes sexuais, escala de auto eficácia sexual. } \\
\text { Não relata a metodologia utilizada. }\end{array}$ & $\begin{array}{l}\text { O conhecimento sexual, as atitudes e a autoeficácia de } \\
\text { recusa dos alunos foram avaliados antes (T0), } \\
\text { imediatamente após (T1) e } 1 \text { mês após a intervenção (T2), } \\
\text { respectivamente. Os alunos do grupo experimental } \\
\text { receberam duas sessões de } 40 \text { minutos do programa } \\
\text { educacional, enquanto o grupo de controle recebeu o modo } \\
\text { usual de educação em saúde sexual e reprodutiva. Em } \\
\text { comparação com o grupo de controle, os alunos do grupo } \\
\text { experimental adquiriram mais conhecimento sexual (p } \\
<.01 \text { ), e desenvolveram atitudes sexuais mais positivas (p } \\
<.05 \text { ) e maior autoeficácia sexual (p <.05) ao longo do } \\
\text { período de estudo. }\end{array}$ \\
\hline
\end{tabular}

Fonte: Autores. 


\section{Resultados e Discussão}

O levantamento bibliográfico possibilitou a identificação de 1.373 estudos, sendo seis na PubMED, oito BVS, 1.309 na Science Direct e 50 na Web of Science. Na triagem, foram excluídos 795 artigos duplicados, permanecendo 578 estudos, dos quais 569 foram excluídos pois não correspondiam a temática e/ou não estavam disponíveis na íntegra, resultando desta forma uma amostra final de 09 artigos (Figura 1).

Quanto aos dados bibliométricos, no que tange ao continente de origem dos estudos de maior ocorrência (n=6) na América do Sul $(67,00 \%)$ seguido de (n=3) na Ásia (33,00\%). Quanto ao nível de evidência em sua maioria (n=5) foram estudos transversais (nível 4) (56,00\%) e logo após, (n=2) respectivamente (22,00\%) relacionados ao relato de experiência (nível 5) e estudo randomizado (nível 1).

Observou-se uma lacuna de estudos primários relacionados à metodologia de educação em saúde principalmente voltada ao público adolescente e de forma especial de prevenção à sífilis. Destacando desta forma, a real necessidade da criação de estratégias de educação em saúde para adolescentes.

Quanto aos resultados não houve um consenso quanto às metodologias utilizadas de educação em saúde para prevenção de sífilis em adolescentes. Porém, a metodologia dos jogos foi a de maior ocorrência em 03 dos 09 estudos. Verificou-se também a real necessidade de traçar ações de educação em saúde para o público adolescente de forma para além da convencional e em conjunto multissetorial envolvendo a escola, professores, saúde e os pais (Tabela1).

O estudo realizado por Andrade et al., (2014) relatou a experiência dos acadêmicos de enfermagem nas atividades realizadas junto aos estudantes de uma escola pública sobre a temática da sífilis, provenientes do projeto de extensão “Aprendendo sobre sífilis: Trabalhando com educação e saúde entre jovens”. A ideia de se trabalhar essa temática se deve ao fato do aumento no número de jovens com vida sexual ativa e de gravidez na adolescência, associado ao elevado número de sífilis congênita em todo país.

O projeto se desenvolveu com adolescentes matriculados no $8^{\circ}$ e $9^{\circ}$ ano do ensino fundamental noturno de uma escola municipal em Cuité, Paraíba, Brasil. Cada turma continha em média 35 alunos, e a faixa etária dos estudantes estava entre 13 a 19 anos de idade. No que diz respeito à forma de abordagem para essas atividades educativas em saúde, no primeiro momento utilizou-se recursos como a apresentação de peças teatrais, que foram elaboradas e interpretadas pelas acadêmicas de enfermagem participantes do projeto, a fim de abordar de maneira mais espontânea e divertida a temática da DST/Sífilis, seu modo de transmissão, diagnóstico, tratamento e prevenção.

Após a apresentação das peças teatrais, os encontros seguintes foram realizados sobre forma de rodas de conversa (duas com cada turma). Como a temática da Sífilis está diretamente relacionada à sexualidade e à outras doenças sexualmente transmissíveis, foram abordados assuntos relativos ao sexo seguro, métodos contraceptivos e AIDS.

Com o andamento do projeto, observou-se que ainda há fragilidade nos serviços de saúde em desenvolverem estratégias que promovam a educação e saúde entre os jovens. Considerou-se também que o profissional que vai trabalhar com esse público específico, precisa de um preparo maior, já que as formas de envolvimento, dinâmica de aprendizado e comportamento são muito peculiares. Outro fato importante observado, é que até mesmo os professores sentiam-se inseguros em abordar temática da DST e sexualidade entre os alunos, mostrando assim, a necessidade de capacitação entre os professores da educação fundamental para que estes possam abordar assuntos mais específicos da adolescência, utilizando estratégias mais apropriadas para o envolvimento desses alunos, criando espaços de diálogo, onde as dúvidas sejam esclarecidas e as informações repassadas com mais segurança.

Resultados similares ao de Wang et al., (2012), Andrade et al., (2014) e como de Dong et al., (2019) e no de Franco (2020) relataram também a experiência da implementação de intervenções educacionais para a promoção da saúde sexual e 
reprodutiva do adolescente escolar por meio de estratégias educacionais ativas e inovadoras. Ao final, destacaram a carência no conhecimento de adolescentes sobre a temática, a intervenção no ambiente escolar mostrou-se um ambiente promissor para o desenvolvimento de conhecimento sobre práticas saudáveis (Franco, 2020).

Xing, Yang, Chow, Zang, (2021) investigaram a eficácia de um programa interativo de educação em saúde sexual e reprodutiva em aspectos de conhecimento, atitudes e auto eficácia entre adolescentes. A amostra foi do tipo por conglomerado estratificado de 469 alunos do ensino médio de duas escolas em uma cidade no leste da China que foram designados para o grupo experimental $(n=233)$ e controle $(n=236)$. Foi avaliado o conhecimento, atitudes e auto eficácia sexual dos adolescentes e foram avaliados antes (T0) e após (T1) um mês após da intervenção (T2).

Os alunos do grupo experimental receberam duas sessões de 40 minutos do programa educacional, enquanto o grupo de controle recebeu o modo usual de educação em saúde sexual e reprodutiva. Observou-se que em comparação com o grupo de controle, os alunos do grupo experimental adquiriram mais conhecimento sexual $(p<0,01)$ e desenvolveram atitudes sexuais mais positivas $(p<0,05)$ e maior auto eficácia sexual $(p<0,05)$ durante o período de estudo.

Considerou-se que a intervenção educacional realizada demonstrou efeitos positivos no conhecimento. A eficácia do conhecimento pode ter sido influenciada por duas condições. Em primeiro lugar, os materiais educacionais foram preparados em linguagem simples e clara, e integrados desenhos animados coloridos e vídeos de animação de uma forma consistente com formatos ou estilos usuais. Em segundo lugar, uma variedade de estratégias participativas (ou seja, jogo de perguntas, alternativamente competição cognitiva de conhecimento, discussões em grupo e ferramentas audiovisuais) no qual atendeu à inclinação dos adolescentes para estímulos de aprendizagem mais dinâmicos e menos abstrato.

O estudo de Wang et al., (2012) analisou o efeito de uma intervenção estrutural abrangente integrada sobre sífilis incidente e infecção pelo HIV em 6 cidades do sul da China, Todas as mulheres com idade $\geq 15$ anos que relataram trocar dinheiro ou presentes por sexo vaginal, oral ou anal com um homem no último ano foram elegíveis para participar.

As mulheres do grupo controle receberam programas de rotina de divulgação da saúde (ou seja, fornecimento de preservativos e materiais de educação em saúde), enquanto o grupo de intervenção também recebeu um pacote abrangente de prevenção estrutural que incluiu o seguinte: promoção da saúde na televisão e no jornal para aumentar a conscientização e comportamentos de busca de saúde; triagem regular de sífilis para aqueles que não fizeram qualquer triagem nos últimos 3 meses através de serviços de divulgação mensal nas configurações para incluir mulheres profissionais do sexo fora da coorte; e maior conexão entre serviços de IST de divulgação e clínica por meio de serviços de referência aprimorados.

Foram detectadas 132 infecções por sífilis incidentes ao longo do estudo, com 109 no grupo controle e 23 no grupo de intervenção. Este estudo demonstra a eficácia e potenciais benefícios para a saúde pública na prevenção e controle da sífilis, fornecendo intervenções estruturais abrangentes expandidas para mulheres profissionais do sexo no sul da China.

Dong et al., (2019) relataram uma intervenção em mulheres trabalhadoras do sexo (FSWs) consideradas como "de baixo custo", de idades variadas (classificadas no estudo como menores de 35 anos e maiores de 35 anos), onde a faixa etária dita como “menores de 35 anos" abrange meninas de 18 e 19 anos, uma vez que no estudo, um dos critérios de inclusão foi ser maior de 18 anos e menor de 60 anos de idade. Os autores relatam sobre o alto risco que estas mulheres tem de adquirir o vírus da imunodeficiência humana (HIV) e infecções sexualmente transmissíveis (ISTs) e de transmiti-los para a população em geral, o que aponta para uma necessidade urgente para desenvolver uma intervenção abrangente de medidas voltadas para mulheres trabalhadoras do sexo de baixa taxa para reduzir infecções por HIV / IST. Um total de 1.024 FSWs elegíveis foram inscritas na pesquisa de linha de base e 843 no acompanhamento. Participantes receberam o pacote de intervenção (incluindo promoção do preservativo, teste de HIV e sífilis, reembolso para custos de tratamento de sífilis e terapia anti-retroviral), e as outras participantes receberam o padrão atual de atendimento. Os resultados mostraram que a infecção por sífilis foi reduzida em 49\% no grupo de intervenção em comparação com o padrão atual do grupo de cuidados ( $\mathrm{P}=0,0378, \mathrm{OR}=0,51,95 \% \mathrm{CI}$ : 0,27-0,96). 
Este estudo demonstrou que intervenções abrangentes podem levar a declínios significativos na infecção por sífilis entre FSWs de baixo nível.

No estudo realizado por Leite, Murray \& Lenz (2015) verificaram que a maior vulnerabilidade desse grupo social (mulheres profissionais do sexo), ocorre com os parceiros íntimos, não-comerciais. Contudo, foi observado que o foco das pesquisas e a forma que são feitas geralmente reforçam a ideia de que a vulnerabilidade decorre de seus clientes. Observou-se também que existem ainda poucos estudos sobre seus contextos de trabalho e fatores estruturais que influenciam práticas sexuais mais seguras com ambos os tipos de parceiros.

Por fim, os autores concluíram sobre a necessidade de ações, políticas e pesquisas que incluam o ambiente e contexto nos quais profissionais do sexo trabalham, que reincorporem o arcabouço de direitos humanos e cidadania, e que as profissionais do sexo devem ser consideradas e tratadas como mulheres, iguais a todas as outras. Assim, as ditas "intervenções estruturais" se tornariam mais efetivas, e consequentemente haveria uma redução nas IST's entre essas mulheres.

Os estudos realizados por Barbosa et al., (2010), Oliveira et al., (2013) e Fonseca et al., (2020) corroboram entre si na estratégia de intervenção e ação educativa. Em ambos os estudos, a metodologia utilizada foram jogos educativos, onde um préteste e um pós- teste foram realizados, antes e após o jogo educativo, com o objetivo de avaliar e estimular o conhecimentos à cerca da sífilis e outras IST’s (transmissão, sintomatologia, prevenção, entre outros) dos adolescentes em questão.

O estudo transversal de Oliveira et al., (2013) investigou o conhecimento de estudantes de escolas públicas (76 estudantes de idades entre 10 a 24 anos), de um Centro de Ensino Médio Integral, na cidade de Bom Jesus - PI, sobre sexualidade, métodos contraceptivos e doenças sexualmente transmissíveis. A coleta dos dados aconteceu em três momentos: primeiro, foi aplicado o questionário (pré-teste); segundo, realizou-se oficinas de prevenção das DST e AIDS ( apresentação de peça teatral; um jogo sobre os métodos contraceptivos; e, ao final, ocorreu uma dinâmica em grupo, em que foram trabalhados os principais questionamentos, apresentados nas ações do projeto, sobre os aspectos do desenvolvimento puberal e as implicações da sexualidade), com a implantação de ações de Educação em Saúde e Orientações sobre as principais DST e suas manifestações clínicas com grupos de até quinze pessoas sobre as temáticas; e, por último, aplicou-se o mesmo questionário (pós-teste) objetivando identificar a diferença de conhecimento dos adolescentes. Evidenciou-se conhecimento deficiente sobre métodos contraceptivos e DST, fato esse que pode torná-los mais vulneráveis em suas práticas sexuais.

O estudo feito por Fonseca et al., (2020) teve como objetivo relatar uma ação educativa por meio de tecnologias educacionais sobre sífilis adquirida para estudantes de uma escola estadual do Pará. O público alvo foram 58 estudantes, sendo 36 homens e 22 mulheres, na faixa etária prevalente de 15 a 17 anos. A ação educativa ocorreu em três momentos: no primeiro momento foi elaborado um folder educativo e distribuído para os alunos, de modo que fosse possível acompanhar a apresentação de uma abordagem geral sobre sífilis. No segundo momento, foi aplicado uma tecnologia adaptada para a realidade dos estudantes elaborada pelos autores, denominado jogo da memória, um jogo didático montado em 14 pares que apresentavam fotos e conceitos sobre o tema abordado, para que os estudantes pudessem reconhecer as figuras e relacionar com os respectivos conceitos. O objetivo do jogo foi promover o reconhecimento através do recurso lúdico, onde o ambiente se torna mais atraente, além de servir de motivação e estimulo para o aprendizado. No terceiro momento, foi utilizado como atividade complementar a dinâmica dos balões onde estimulou-se uma competição de conhecimentos.

O estudo revelou um conhecimento superficial dos alunos sobre sífilis como infecção sexualmente transmissível, assim como, sua prevenção e a utilização de teste rápido para diagnóstico. As principais e mais frequentes dúvidas sobre sífilis estavam voltadas para sua transmissão, diagnóstico e prevenção. Apesar disso, os participantes já tinham ouvido falar da doença e sabiam que esta possui cura. Assim sendo, a atividade permitiu a diminuição da vulnerabilidade desses indivíduos diante da sífilis e a todos os seus agravos. No final da ação educativa baseada nas dinâmicas utilizadas, foi possível perceber a aquisição de 
conhecimentos sobre a sífilis pelos estudantes, podendo ser multiplicadores de informações para seus pares, reforçando desta forma a relevância da educação em saúde através de tecnologias educacionais destinadas aos adolescentes.

Barbosa et al., (2010) realizaram um estudo exploratório descritivo em uma escola pública de Fortaleza-CE, com 85 adolescentes na faixa etária de 14 a 19 anos, onde foi abordado a prevenção de DST/AIDS, com a finalidade de expor a utilização de jogos educativos como forma de educação em saúde para o público adolescente.

Como atividade educativa, foi usado um jogo semelhante ao dominó, contendo 30 peças com indagações e respostas que abordavam assuntos correlacionados à sexualidade dos adolescentes, como: conceitos da anatomia feminina e masculina, puberdade e adolescência, sexo/sexualidade, DST/HIV/AIDS, gravidez e métodos de prevenção. Inicialmente aplicou-se o préteste, que continha por nove questões de múltipla escolha, com o intuito de estimar o conhecimento dos adolescentes antes da realização do encontro educativo. Posterior a aplicação do jogo educativo, foram aplicados os formulários de pós-teste, que continham questões iguais às do pré-teste, de modo a verificar o conhecimento adquirido através do uso do jogo.

Foi relatado que, no que se refere às pessoas com maiores possibilidades de contrair uma DST ou vírus HIV, os adolescentes apresentam dificuldade de compreensão relacionadas ao fato de que o risco de adquirir qualquer DST caso seu parceiro esteja infectado é para qualquer pessoa que não se proteja utilizando preservativo durante as relações sexuais. No pensamento dos adolescentes, a ideia de que os homossexuais e os profissionais do sexo apresentam maior vulnerabilidade à se infectarem pelo vírus HIV e às DST's, é predominante.

Os resultados evidenciaram que os alunos, em sua maioria, assimilaram as questões discutidas pelo grupo, embora não seja possível assumir uma mudança de comportamento. Uma vez que, o comportamento sexual seguro é consequência do nível de conhecimento aliado ao contexto cultural onde o indivíduo encontra-se e às crenças apresentadas por ele.

Concluiu-se que a utilização de um jogo educativo como técnica de educação em saúde para adolescentes na prevenção de DST/AIDS foi uma prática bem sucedida, pois favoreceu o desempenho do fenômeno educativo através da junção entre informação, debate, reflexão, influência recíproca e participação em grupo. Os adolescentes tiveram a oportunidade de sanar as dúvidas, ocupar espaços do conhecimento relacionados a sexualidade e prevenção de DST e AIDS, e exercer interação de maneira desinibida, favorecendo a atuação de todos no processo de aprendizado.

Em um estudo de investigação que avaliou as condutas de risco para infecções sexualmente transmissíveis (ISTs) em estudantes do ensino médio, descreveu em seus achados que relacionado ao conhecimento sobre manifestações de ISTs, $62,4 \%$ informaram dor na região genital como sinal de alerta, todavia a presença de feridas e corrimento foi reconhecida por menos de $40 \%$ dos adolescentes. Os adolescentes, em sua maioria, já receberam orientações sobre sexualidade e afirmaram possuir conhecimento sobre ela, porém os resultados demonstram falha no entendimento (Rizzon et al., 2021).

No estudo analítico sobre a elaboração do jogo on-line Papo Reto, realizado por Souza, et al., (2016), destinado a adolescentes de 15 a 18 anos, teve como resultados as interações de 60 adolescentes de Belo Horizonte e de São Paulo. Exemplos das potencialidades do jogo foram constituídas para favorecer a abordagem sobre sexualidade com adolescentes por meio da simulação de realidades, da invenção e da interação. Com base nessas potencialidades, foram discutidas quatro categorias reflexivas: jogo como dispositivo pedagógico; como simulação de realidades; como dispositivo para a aprendizagem inventiva; e como o jogo potencializa a interação. Foi possível concluir que o jogo, ao permitir que os adolescentes se arrisquem por novos caminhos, possibilita que se tornem mais criativos e ativos na produção de sentidos, na criação de seus discursos e nas formas de pensar, sentir e agir no campo da sexualidade.

O estudo do tipo pesquisa-ação, de abordagem qualitativa de Gubert et al., (2009), teve a intenção de abordar o processo de educação em saúde através da utilização de tecnologias educativas junto a adolescentes no cenário escolar. O estudo foi realizado em uma escola pública municipal localizada em Fortaleza-CE, com uma turma de 30 alunos, com idades entre 14 e 18 anos, cursando o segundo ano do ensino médio. As oficinas tiveram o objetivo de promoção da reflexão/ação juntamente com 
os participantes sobre as temas: sexualidade, gênero, DST/AIDS e métodos contraceptivos. Através dos resultados foi possível perceber brechas no conhecimento relacionadas às maneiras de adquirir as DST's e uso correto de métodos contraceptivos. Outro quesito levantado faz referência às questões de gênero que aparentam influir na qualidade de vida dos jovens estudados.

A utilização de recursos educativos pôde atiçar entre os adolescentes, uma reflexão relacionada a vivência da saúde sexual e reprodutiva a partir das fragilidades detectadas. O trabalho se deu a partir do processo dialético entre o diálogo e elaboração das vivências referentes à sexualidade. A primeira fase do estudo caracterizou-se pela observação e escuta dos adolescentes através de uma roda de conversa para se obter uma visão do interesse do grupo e apresentação dos objetivos do Projeto. Por conseguinte, os adolescentes tiveram um debate sobre os temas que mais lhe interessavam, voltados à Saúde Sexual e Reprodutiva. Em seguida, foram desenvolvidas as oficinas educativas apoiadas no Círculo de Cultura, abordando as temáticas escolhidas pelos adolescentes. A realização das oficinas deu-se através da Análise da Demanda - Levantamento; Pré-análise da Problemática do Contexto e do Grupo - Planejamento; Levantamento dos Temas-Geradores e Definição do Foco - Execução e Avaliação.

O propósito foi de despertar os jovens a uma reflexão crítica e problematizadora, permitindo que os saberes individuais fossem coletivizados numa experiência comum. Para discussão sobre as DST, foram apresentados folderes veiculados pelo Programa Nacional de DST/AIDS, contendo informações sobre as doenças e formas de contágio, além de álbum seriado ilustrado. Tais materiais, em conjunto com as informações discutidas pelo grupo, favoreceram o processo de comunicação entre os adolescentes e as pesquisadoras acerca de DST e AIDS. Foi reforçado no estudo, a necessidade do Enfermeiro em produzir novas tecnologias educacionais, extrapolando as atividades de educação em saúde baseada em ações pontuais e que não reconhecem as verdadeiras necessidades, desejos e aspirações de seus integrantes.

Na revisão integrativa realizada por Barreto et al, (2016) notou o enfoque de produções que abordavam as ações de educação em saúde, no que diz respeito à saúde sexual do adolescente, preferencialmente, em âmbito escolar. E, foi relatado pelos autores que, de acordo com a literatura consultada, este é considerado um ambiente favorável e privilegiado para efetivar ações de promoção da saúde, em razão da sua capilaridade e abrangência, contribuindo para a elaboração de valores pessoais, crenças, conceitos e maneiras de conhecer o mundo, influenciando diretamente na produção social da saúde para a concretização de ações de promoção da saúde voltadas para o fortalecimento das capacidades dos indivíduos para a tomada de decisão adequada à sua saúde e à comunidade. Contudo, direcionar as ações de promoção da saúde ao adolescente tendo a escola como um campo prioritário pode ser um fator limitante da atuação dos profissionais de saúde em outros contextos políticos e sociais.

Beserra et al., (2008) realizaram uma pesquisa qualitativa com objetivo de investigar a sexualidade das adolescentes a partir da ação educativa do enfermeiro na prevenção de doenças sexualmente transmissíveis que foi realizada em uma escola pública em Fortaleza-CE com 10 meninas entre 14 e 16 anos.

Adotou-se como método o Círculo de Cultura, que foi formado por cinco encontros com duração de cinquenta minutos, abordando os temas: adolescência, sexualidade, doenças sexualmente transmissíveis, vida sexual segura e uso do preservativo. O primeiro encontro foi constituído por palavras geradoras, que são ditas pelos próprios participantes do grupo. Essas palavras significam o contexto real no qual os educandos vivem, sendo essenciais para a condução do Círculo de Cultura e para a realização dos encontros posteriores. O segundo encontro envolveu a integração das palavras geradoras, quando se dialogaram temas do interesse das jovens. No terceiro encontro houve abordagem das doenças sexualmente transmissíveis. No quarto encontro, ocorreram discussões diversas, refletindo-se sobre temas que contemplavam a saúde sexual e reprodutiva das adolescentes. O quinto encontro focou a avaliação do processo.

Observou-se que as meninas associam o sexo à sexualidade de forma predominante e que tinham pouca compreensão das vulnerabilidades que estavam expostas numa prática sexual desprotegida. Evidenciou-se que a execução do Círculo de 
Cultura permitiu que as meninas explorassem e discutissem sobre diversos temas que englobavam sua sexualidade, e que era um momento para ações de educação em saúde com a meta de isentá-las de riscos.

O estudo descritivo, do tipo relato de experiência, realizado por Monteiro \& Vieira (2015), com adolescentes escolares de uma escola pública localizada em Recife/PE, teve como objetivo, relatar a experiência na realização de atividades de educação em saúde escolar, também utilizando a metodologia de Círculos de Cultura de Paulo Freire sobre as IST's e sexo seguro. A atividade despertou responsabilidade social dos adolescentes com as questões de sua saúde na prevenção e controle das IST's. Eles demonstraram interesse, participando e questionando sobre os assuntos em questão. As autoras relatam que foi observado um resultado positivo e foi verificado o domínio do conteúdo pelos escolares. Concluíram que, por meio da metodologia do Círculo de Cultura, novos conhecimentos sobre as temáticas foram adquiridos e mesmo que não gerem uma mudança de comportamento imediata, podem contribuir para um repensar de suas práticas e atitudes para o futuro.

Já a pesquisa realizada por Vieira et al., (2021) objetivou identificar a prevalência do início da atividade sexual em adolescentes e a prática de sexo seguro entre eles, destacou que a idade média da sexarca foi de 14,1 anos, e houve a tendência de iniciação sexual precoce no sexo masculino. Um terço das primeiras relações sexuais foram desprotegidas (33,9\%). As participantes do sexo feminino apresentavam maior conhecimento a respeito de contracepção e prevenção de doenças, contudo, menor adesão ao uso de preservativos e maior utilização de contraceptivos orais e de emergência.

\section{Considerações Finais}

Através deste estudo, percebeu-se que são poucos os estudos com a temática que envolve a educação em saúde na prevenção de sífilis em adolescentes. Essa observação é importante, pois a fase de vida em que se encontra o indivíduo influencia diretamente na experiência, vivência e no processo ensino-aprendizagem dos participantes das atividades educativas.

Não houve um consenso relativo as estratégias utilizadas. Porém, a de maior ocorrência foi por meio dos jogos educativos como estratégia de educação em saúde na prevenção de sífilis em adolescentes.

Como limitação desse estudo, observou-se ausência de estudos primários nessa temática e público envolvido. Destacando-se desta forma, a real necessidade no desenvolvimento e aplicação prática de estratégias de educação em saúde na prevenção de sífilis na adolescência. Observou-se também que o ambiente escolar mostrou-se como mediador chave importante na educação sexual e prevenção de IST's em adolescentes.

Conclui-se então que há necessidade de mais estudos no mundo para fornecer mais informações sobre a temática abordada, principalmente trabalhos realizados em países em desenvolvimento como os da América Latina, Ásia e África. Dentre esses, os estudos com ensaios clínicos randomizados são muito necessários pois oferecem um aporte mais consistente em evidências científicas. São imprescindíveis trabalhos que tratem especificamente sobre a população adolescente, pois esta possui especificidades bem definidas da própria faixa etária, muito distintas da população adulta. Necessita-se que essa população adolescente possa ser estudada e discutida considerando suas especificidades. É muito importante que esses novos estudos também trabalhem mais com desenhos metodológicos de nível de evidência científica mais elevado, pois a comprovação nesses trabalhos é muito mais fidedigna. Além da necessidade de desenvolvimento e aplicação prática dessas estratégias em localidades assoladas por casos de sífilis na adolescência, estreitando a cooperação de países desenvolvidos com os países não desenvolvidos, principalmente a África. 


\section{Referências}

Aljoharah, A., Almuneef, M., \& Minhas, H. R. (2012). Knowledge, attitudes, and resources of sex education among female adolescents in public and private schools in Central Saudi Arabia. Saudi Med J., 33(9): 1001-1009.

Almeida, R. A. S., Corrêa, R. G. C. F., Rolim, I. L. T. P., Hora, J. M., Linard, A. G., et al. Conhecimento de adolescentes relacionados às doenças sexualmente transmissíveis e gravidez. REBEn. 70 (5). https://www.scielo.br/j/reben/a/p4gD43L6gJhMZv3yGkRfvnM/?format=pdf\&lang=pt.

Andrade, L. D. F., Farias, K. E. E., Araújo, G. H., Costa, G. O. M., Nunes, P. C., \& Saraiva, A. M. (2014). Promovendo ações educativas sobre sífilis entre estudantes de uma escola pública: relato de experiencia. Rev Bras de Ciências da Saúde, 18(2). doi:10.4034/RBCS.2014.18.02.10.

Barbosa, S. M., Dias, F. L. A., Pinheiro, A. K. B., Pinheiro, P. N. C., \& Vieira, N. F. C. (2010). Jogo educativo como estratégia de educação em saúde para adolescentes na prevenção às DST/AIDS. Rev. Eletr. Enf., 12(2): 337-41. 10.5216/ree.v12i2.6710.

Barreto, R. M. A.,, Cavalcante, A. S. P., Mira, Q. L. M., Vasconcelos, M. I. O. V., \& Brito, M. C. C. (2016) Ações Educativas em Saúde para o Público Adolescente: Uma Revisão Integrativa. Rev. APS, 19(2): 277 - 285. Disponível em: https://periodicos.ufjf.br/index.php/aps/article/view/15583/8172.

Beserra, E. P., Pinheiro, P. N. C., \& Barroso, M. G. T. (2008). Ação educativa do enfermeiro na prevenção de doenças sexualmente transmissíveis: uma investigação a partir das adolescentes. Esc Anna Nery Rev Enferm., 12(3):522-28. https://doi.org/10.1590/S1414-81452008000300019.

Brasil (2014). Diretrizes Metodológicas. Elaboração de revisão sistemática e metanálise de estudos observacionais comparativos sobre fatores de risco e prognóstico. Ministério da Saúde. Secretaria de Ciência, Tecnologia e Insumos Estratégicos Departamento de Ciência e Tecnologia.

Brasil. Ministério da Saúde (2017). Agenda de ações estratégicas para redução da sífilis. Secretaria de Vigilância em Saúde. Departamento de Vigilância, Prevenção e Controle das Infecções Sexualmente Transmissíveis, do HIV/Aids e das Hepatites Virais. http://www.aids.gov.br/pt-br/pub/2017/agenda-de-acoesestrategicas-para-reducao-da-sifilis-no-brasil.

Brasil. Ministério da Saúde (2018). Proteger e cuidar da saúde de adolescentes na atenção básica. $2^{\mathrm{a}}$ edição. Ministério da Saúde. Secretaria de Atenção à Saúde Departamento de Ações Programáticas e Estratégicas Coordenação-Geral de Saúde do Adolescente e do Jovem. https://bvsms.saude.gov.br/bvs/publicacoes/proteger_cuidar_adolescentes_atencao_basica_2ed.pdf.

Brasil. Ministério da Saúde (2020). Boletim Epidemiológico. Secretaria de Vigilância em Saúde. Número especial. Out 2020. http://www.aids.gov.br/ptbr/pub/2020/boletim-sifilis-2020.

Carneiro, R. F., Silva, N. C., Alves, T. A., \& Alburquerque, D. O., Brito, D. C., et al. (2015). Educação sexual na adolescência: uma abordagem no contexto escolar. Sanare, 14 (1).

Carvalho, R. X. C., \& Araújo, T. M. E. (2020). Knowledge, attitudes and practices of university adolescents about syphilis: a cross-sectional study in the Northeast. Revista de Saúde Pública, 54(120). https://doi.org/10.11606/s1518-8787.2020054002381.

Dong, W., Zhou, C., Rou, Ke-Ming, Wu, Zun-You, Chen, J., et al. (2019). A community-based comprehensive intervention to reduce syphilis infection among low-fee female sex workers in China: a matched-pair, community-based randomized study. Infectious Diseases of Poverty, 8(97). https://doi.org/10.1186/s40249019-0611-z.

Fonseca, A. C. M., Batista, A. B. G., Santos, A. P., Leão, E. C., \& Garcia, G. K. C. S. (2020). Inovações tecnológicas na abordagem de sífilis adquirida na adolescência para estudantes de uma escola estadual do Pará: um relato de experiência. Electronic Journal Collection Health, 41. https://doi.org/10.25248/reas.e2234.2020.

Franco, M., Barreto, M., Carvalho, J., Silva, P., Moreiras, W., et al. (2020). Educação em saúde sexual e reprodutiva do adolescente escolar. Revista de Enfermagem UFPE, Jul 3. Disponível em: https://periodicos.ufpe.br/revistas/revistaenfermagem/article/view/244493

Gubert, F. A., Santos, A. C. L., Aragão, K. A., Pereira, D. C. R., Vieira, N. F. C., \& Pinheiro, P. N. C. (2009). Tecnologias educativas no contexto escolar: estratégia de educação em saúde em escola pública de Fortaleza-CE. Rev. Eletr. Enf., 11(1):165-72. http://www.fen.ufg.br/revista/v11/n1/v11n1a21.htm.

Leite, G.S., Murray, L., \& Lenz, F. O Par e o Ímpar: o potencial de gestão de risco para a prevenção de DST/HIV/AIDS em contextos de prostituição. (2015). Rv Bras Epidemiol., 18(01): 07-25. 10.1590/1809-4503201500050003.

Liberati, A., Moher D., Tetzlaff. J, \& Altman, D. G. PRISMA Group (2009). Preferred reporting items for systematic reviews and meta-Analyses: the PRISMA statement. PLOS Medicine, 339. https://doi.org/10.1136/bmj.b2700.

Luppi, C. G., Gomes, S. E. C., Silva, R. J. C., Ueno, A. M., Santos, A. M. K., et al. (2018). Fatores associados à coinfecção por HIV em casos de sífilis adquirida notificados em um Centro de Referência de Doenças Sexualmente Transmissíveis e Aids no município de São Paulo, 2014. Epidemiologia e Serviços de saúde, 27 (1). https://doi.org/10.5123/S1679-49742018000100008.

Monteiro, E. M. L. M., \& Vieira, N. F. C. Educação em saúde a partir de círculos de cultura. (2010). Rev Bras. Enferm. 6(3). https://doi.org/10.1590/S003471672010000300008

Oliveira, K. N. S., Oliveira, K. N. S., Bezerra, M. A. R., Rocha, R. C., Santos, L. R., \& Saraiva, P. V. (2013). Educação sexual na adolescência e juventude: abordando as implicações da sexualidade no contexto escolar. Sanare, 12(2):7-13.

Ouzzani, M., Hammady, H., Fredorowicz, Z., \& Elmagarmid, A. (2016) Rayyan-a web and mobile app for systematic reviews. Syst Rev. 5: 210. https://doi.org/10.1186/s13643-016-0384-4.

Rayyan (2021). https://rayyan.qcri.org. 
Research, Society and Development, v. 10, n. 12, e545101220577, 2021

(CC BY 4.0) | ISSN 2525-3409 | DOI: http://dx.doi.org/10.33448/rsd-v10i12.20577

Rizzon, B. B., Souza, V. B., Madeira, K., Machado, L. V., \& Magalhães, M. (2021). Comportamento de risco para infecções sexualmente transmissíveis em estudantes do ensino médio. Femina., 49(1): 52-57.

Santos, M. M. D., Rosendo, T. M. S. D. S., Lopes, A. K. B., Roncalli, A. G., \& Lima, K. C. D. (2021) As fragilidades na atenção primária à saúde favorecem o crescimento da sífilis adquirida. PLoS Negl Trop Dis. 15(2): e0009085. https://doi.org/10.1371/journal.pntd.0009085.

Santos, S. B., Machado, A. P. A., Sampaio, L. A., Abreu, L. C., \& Bezerra, I. M. P. (2019). Acquired Syphilis: construction and validation of educational technology for adolescents. J Hum Growth Dev., 29(1): 65-74.http://dx.doi.org/10.7322/jhgd.157752.

Souza, V., Gazzinelli M. F., Soares, A. N., Fernandes, M. M., Oliveira, R. N. G., \& Fonseca, R. M. G. S. (2017). O jogo como estratégia para abordagem da sexualidade com adolescentes: reflexões teórico-metodológicas. Rev. Bras. Enferm, 70(2): 394-401.

Vieira, K. J., Barbosa, N. G., Dionízio, L. A., Santarato, N., Monteiro, J. C. S., \& Gomes-Sponholz, F. A. (2021). Início da atividade sexual e sexo protegido em adolescentes. Esc. Anna Nery, 25(3): e20200066. http://www.revenf.bvs.br/scielo.php?script=sci_arttext\&pid=S1414-81452021000300212\&lng=pt.

Wang, B., Wang, Qian-Qiu, Yin, Yue-Ping, Liang, Guo-Jun, Jiang, N. et al. (2012). The Effect of a Structural Intervention for Syphilis Control Among 3597 Female Sex Workers: A Demonstration Study in South China. 10.1093/infdis/jis466.

World Health Organization (2016). Who guidelines for the Treatment of Treponema pallidum (syphilis). https://apps.who.int/iris/bitstream/handle/10665/249572/9789241549806-eng.pdf;jsessionid=47A2239BD0ABE3ECA1958CC66DB37801?sequence=1.

World Health Organization. $138^{\text {th }}$ session provisional agenda item 9.2. Draft global health sector strategies. Sexually transmitted infections, 2016-2021 [internet]. Geneva: World Health Organization; 2015. http://apps.who.int/gb/ebwha/pdf_files/EB138/B138_31-en.pdf.

Xing, Ma R. N., Yuanyuan, Yang R. N., Mmed, B. S. N., Ka Ming, C. R. N., et al. (2021). Chinese adolescents' sexual and reproductive health education: A quasi-experimental study. Public Health Nurs, 1(10). 10.1111/phn.12914. 